\title{
Analysis of the blurring in stencil lithography
}

\author{
O Vazquez-Mena ${ }^{1,3}$, L G Villanueva ${ }^{1}$, V Savu $^{1}$, K Sidler $^{1}$, \\ P Langlet $^{2}$ and J Brugger ${ }^{1,3}$ \\ ${ }^{1}$ Microsystems Laboratory, Ecole Polytechnique Fédérale de Lausanne (EPFL), 1015, \\ Lausanne, Switzerland \\ ${ }^{2}$ Center of Micro-Nano-Technology, EPFL, Switzerland \\ E-mail: oscar.vazquez@epfl.ch and juergen.brugger@epfl.ch
}

Received 26 July 2009, in final form 2 September 2009

Published 18 September 2009

Online at stacks.iop.org/Nano/20/415303

\begin{abstract}
A quantitative analysis of blurring and its dependence on the stencil-substrate gap and the deposition parameters in stencil lithography, a high resolution shadow mask technique, is presented. The blurring is manifested in two ways: first, the structure directly deposited on the substrate is larger than the stencil aperture due to geometrical factors, and second, a halo of material is formed surrounding the deposited structure, presumably due to surface diffusion. The blurring is studied as a function of the gap using dedicated stencils that allow a controlled variation of the gap. Our results show a linear relationship between the gap and the blurring of the directly deposited structure. In our configuration, with a material source of $\sim 5 \mathrm{~mm}$ and a source-substrate distance of $1 \mathrm{~m}$, we find that a gap size of $\sim 10 \mu \mathrm{m}$ enlarges the directly deposited structures by $\sim 50 \mathrm{~nm}$. The measured halo varies from 0.2 to $3 \mu \mathrm{m}$ in width depending on the gap, the stencil aperture size and other deposition parameters. We also show that the blurring can be reduced by decreasing the nominal deposition thickness, the deposition rate and the substrate temperature.
\end{abstract}

S] Supplementary data are available from stacks.iop.org/Nano/20/415303

(Some figures in this article are in colour only in the electronic version)

\section{Introduction}

Stencil lithography (SL) is a high resolution shadow mask technique [1-3] that allows the patterning of structures and devices such as metallic nanodots [2, 4], nanowires [5-7] and NEMS [8]. Compared to other techniques, like electron beam, nano-imprint and deep UV lithography, SL has the important advantage of not requiring any resist processing, such as coatings, chemical solvents or energy radiation. This allows using SL on a broader range of substrates compared to resist-based techniques and reducing the steps required for patterning. For instance, SL has been used for patterning substrates like self-assembled monolayers [9], organic layers [10], polymer substrates [11], CMOS devices [8], cantilevers and non-planar substrates [3]. Recently, stencils have also been used as masks for dry etching and ion implantation [12-14].

\footnotetext{
3 Authors to whom any correspondence should be addressed.
}

An important feature of stencil lithography is that the stencil and the substrate are not in contact. The gap between the stencil and the substrate is due to several factors, like wafer curvature, membrane stress and any topography on the substrate. The gap and the absence of resist processing allow using stencil lithography on chemically or mechanically fragile substrates that can be damaged by radiation, solvents or mechanical pressure. It also allows the patterning on high topography structures where resist coating is difficult. However, the gap also produces a blurring in the deposited structures, reducing the resolution of SL [1, 15-18]. The blurring is also affected by the properties of the deposited material and the substrate $[18,5]$. To our knowledge, the characteristics and the quantitative dependence of the blurring on the stencil-substrate gap and other parameters have not been established experimentally. In this sense, an understanding of the blurring behavior is important to improve the resolution and to determine the limiting factors in stencil lithography. 


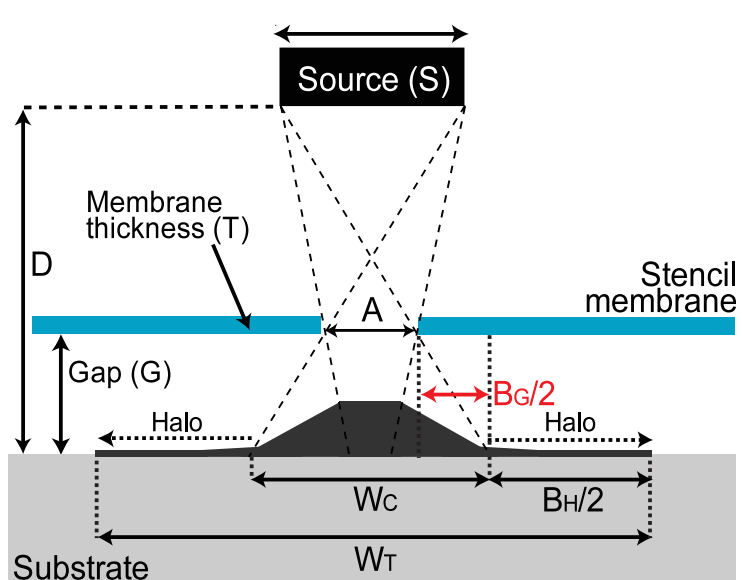

Figure 1. Blurring description. Diagram showing the role of geometry and material spreading in the blurring in stencil lithography. The structure consists of a central structure $\left(W_{\mathrm{C}}\right)$ surrounded by a halo of material due to material spreading. This diagram shows the different elements affecting the blurring: source-substrate distance $(D)$, material source size $(S)$, stencil-substrate gap $(G)$, stencil aperture width $(A)$ and membrane thickness $(T)$. It also shows the width of the central structure $\left(W_{\mathrm{C}}\right)$, the width of the total structure including the halo $\left(W_{\mathrm{T}}\right)$, the blurring due to the geometry $\left(B_{\mathrm{G}}=W_{\mathrm{C}}-A\right)$ and the blurring due to the halo $\left(B_{\mathrm{H}}=W_{\mathrm{T}}-W_{\mathrm{C}}\right)$. (Diagram not to scale.)

In this work, firstly, the blurring in structures deposited by SL is described. Then, dedicated stencils designed to have a controlled variation of the gap in a single deposition are presented. Using these stencils, the blurring is studied as a function of the stencil-substrate gap for Al depositions by ebeam evaporation. Then, the effect of the nominal deposition thickness, deposition rate and substrate temperature on the blurring is also analyzed. Finally, other metals (Au, Pt, Ti and $\mathrm{Cr}$ ) are also deposited through stencils, showing a blurring similar to that observed for $\mathrm{Al}$ depositions. These results are discussed and different mechanisms affecting the blurring are proposed.
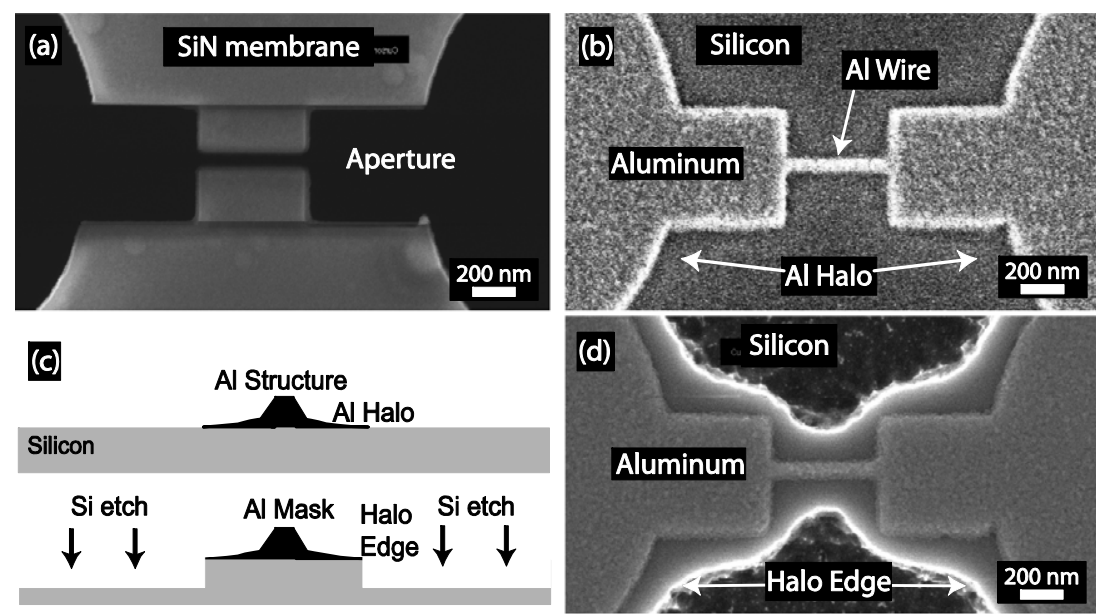

Figure 2. Halo and contrast etching. (a) SEM image of a stencil aperture and (b) its corresponding deposited Al structure on a silicon substrate showing the clear correspondence between them. In (b), the halo surrounding the deposited structure is hardly visible. (c) Scheme of the silicon 'contrast etching' used to enhance the contrast of the halo. (d) SEM image of the same structure in (b), after the contrast etching, revealing clearly the halo around the central structure. 
(a)
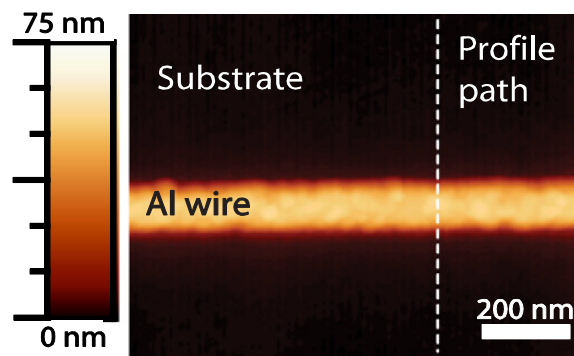

(b)
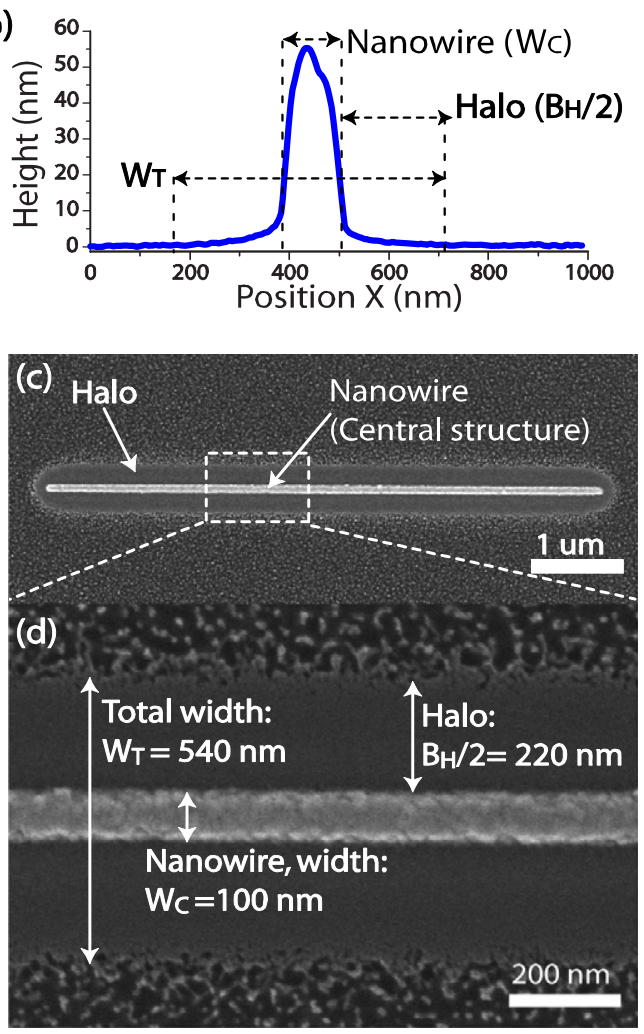

Figure 3. Central structure and halo. (a) TM AFM image before the contrast etching of an $\mathrm{Al}$ nanowire deposited through a nanoslit aperture $90 \mathrm{~nm}$ wide. (b) TM AFM profile of the deposited structure from (a), showing a thickness of $55 \mathrm{~nm}$. (c) SEM image of the nanowire after the contrast etching and the halo surrounding it.

(d) Close-up showing the width of the nanowire, $W_{\mathrm{C}}=100 \mathrm{~nm}$, and of the surrounding halo, $B_{\mathrm{H}}=440 \mathrm{~nm}$. Comparing (b) and (d), the thickness of the halo can be estimated as $\lesssim 5 \mathrm{~nm}$.

in a $100 \mathrm{~nm}$ thick low stress silicon nitride (LS SiN) stencil membrane. Figures 3(a) and (b) show the image and the profile of the $\mathrm{Al}$ nanowire by tapping mode atomic force microscopy (TM AFM) showing a thickness of $\sim 55 \mathrm{~nm}$. Since the halo is difficult to resolve with AFM and SEM, the contrast etching described before was performed. Figures 3(c) and (d) show SEM images of the nanowire with a width of $W_{\mathrm{C}}=100 \mathrm{~nm}$ and the total structure including the halo of $W_{\mathrm{T}} \sim 540 \mathrm{~nm}$. From the TM AFM profile of the structure, the estimated thickness of the halo is $<5 \mathrm{~nm}$.

To analyze the blurring, it will be assumed that the width of the central structure $\left(W_{\mathrm{C}}\right)$ is determined by the aperture size $(A)$ plus the size enlargement of the structure due to the geometry of the source-stencil-substrate configuration. The width of the total structure $\left(W_{\mathrm{T}}\right)$ will be determined by the
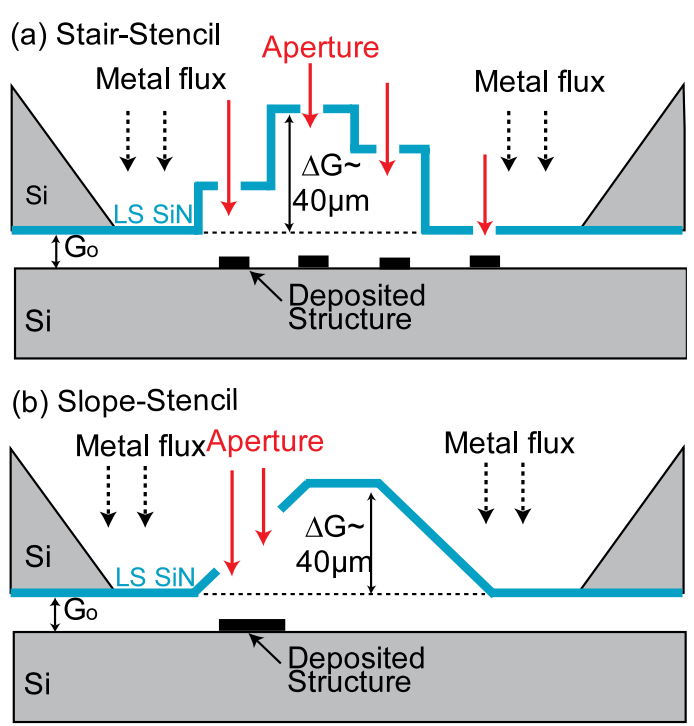

Figure 4. Stencils for controlled variation of the gap. (a) Stair-stencil containing terraces separated vertically by $\sim 5 \mu \mathrm{m}$, allowing different gaps. In total there are seven terraces, not shown in the diagram. (b) Slope-stencil for which the gap is continuously changed with a $54^{\circ}$ slope. When fixing the stencils on top of a substrate, there is already a gap $G_{o}$ between the chip and the substrate which is normally $<5 \mu \mathrm{m}$. (Diagrams not to scale.)

width of the central structure $\left(W_{\mathrm{C}}\right)$ plus the halo due to material spreading. From these assumptions, we will define two types of blurring, the geometrical blurring, $B_{\mathrm{G}}$, and the halo-blurring, $B_{\mathrm{H}}$, as follows (see figure 1):

$$
B_{\mathrm{G}}=W_{\mathrm{C}}-A \quad B_{\mathrm{H}}=W_{\mathrm{T}}-W_{\mathrm{C}}=W_{\mathrm{T}}-B_{\mathrm{G}}-A .
$$

From a geometrical analysis, $B_{\mathrm{G}}$ can be modeled as:

$$
B_{\mathrm{G}}=\frac{G(S+A)+D A-S T / 2}{D+T / 2}-A .
$$

This expression can be approximated and simplified to $[1,15]$ :

$$
B_{\mathrm{G}} \approx \frac{G S}{D}
$$

if $D \gg T, G \gg T$ and $S \gg A$, which is satisfied in depositions with stencil membranes $\sim 100 \mathrm{~nm}$ thick. Concerning the halo, currently there is no analytical model to estimate the size of $B_{\mathrm{H}}$.

\subsection{Stencils for a controlled variation of the gap}

In order to study the effect of the gap on the blurring, two kinds of stencils that allow a controlled variation of the gap in a single deposition were fabricated. One type consists of 'stair-stencils' containing membranes with steps and terraces at different heights and with apertures on the different terraces as shown in figure 4(a). The vertical distance between the terraces is $\sim 5 \mu \mathrm{m}$. The second type consists of 'slope-stencils' containing membranes with a slope of $54^{\circ}$. These stencils have nanoslit apertures going down through the sloped membranes (long axis of slits parallel to the slope direction) as shown 
in figure 4(b). This allows depositions through the same aperture with a varying gap. In both cases, the membranes are made of LS SiN $100 \mathrm{~nm}$ thick. The membrane height profile was measured by optical profilometry. The variation in vertical distance between the top and bottom of the membranes is $\sim 40 \mu \mathrm{m}$. The apertures in the stencil membranes were fabricated with focused ion beam milling and consist of slits with widths from $100 \mathrm{~nm}$ to $2 \mu \mathrm{m}$. In the case of the stairstencils the slits are $5 \mu \mathrm{m}$ long, whereas for the slope-stencils, the length of the slits projected on the substrates is $\sim 25 \mu \mathrm{m}$. The fabrication process and the fabricated stencils are shown in the supporting information in figure SI 1 (available at stacks.iop.org/Nano/20/415303). The stencils are chips of $2 \times 2 \mathrm{~cm}^{2}$ in size and were fixed to the substrate by adhesive tape. As shown in figure 4, even between the flat part of the membranes and the substrate there is already an initial gap, marked as $G_{o}$. This $G_{o}$ gives an offset that has to be added to the gap from the membrane profile $(\Delta G)$ to obtain the total substrate-stencil gap $\left(G=G_{o}+\Delta G\right)$. $G_{o}$ was measured after all performed depositions and it was usually $\lesssim 5 \mu \mathrm{m}$. In depositions using $100 \mathrm{~mm}$ diameter full wafer stencils and substrates, $G_{o}$ can increase up to $10-30 \mu \mathrm{m}$ due mainly to the curvature of the wafers.

\subsection{Blurring as a function of gap}

To study the blurring as function of the gap, a $60 \mathrm{~nm}$ thick aluminum deposition was performed by e-beam PVD through a stair-stencil on a silicon substrate. The depositions were performed at a base pressure of $10^{-6}$ mbar, a deposition rate of $4 \AA \mathrm{s}^{-1}$, a source-substrate distance $D=1 \mathrm{~m}$ and at room temperature. The electron beam that heats the material source is $\sim 5 \mathrm{~mm}$ in diameter and is static without any sweeping on the material source. The profile and thickness of the deposited structures were obtained by TM AFM analysis before the contrast etching [19]. The widths of the stencil apertures $(A)$ and of the deposited structures $\left(W_{\mathrm{C}}\right.$ and $\left.W_{\mathrm{T}}\right)$ were obtained by SEM analysis after performing a contrast etching. The gap effect on the blurring is illustrated in figure 5, showing three aluminum structures deposited through $200 \mathrm{~nm}$ wide slit apertures located at different terraces on the stencil membranes, i.e. having different gaps with the substrate. A $\Delta G=0$ corresponds to apertures located in the bottom part of the stencils, with a total gap $G=G_{o}$. From figures 5(a) to (c), it is observed that the halo becomes larger as the gap increases. Similarly, the close-up images from figures 5(d) to (f) show how the central structure becomes more blurred when the gap increases. This is confirmed by the TM AFM profiles shown in figure $5(\mathrm{~g})$ showing how the thickness of the structures decreases as the gap increases.

The results for the different aperture sizes and gaps are summarized in figure 6 . The measured values of $B_{\mathrm{G}}$ are plotted as a function of $\Delta G$ in figure 6(a). The plotted values are obtained averaging $B_{\mathrm{G}}$ from structures deposited through apertures of different sizes at a constant $\Delta G$. The results show an approximate linear behavior of $B_{\mathrm{G}}$ as a function of $\Delta G$, as expected from equation (3). After performing a linear fitting of these results to $B_{\mathrm{G}}=\alpha(\Delta G)+\beta$, the obtained coefficients are
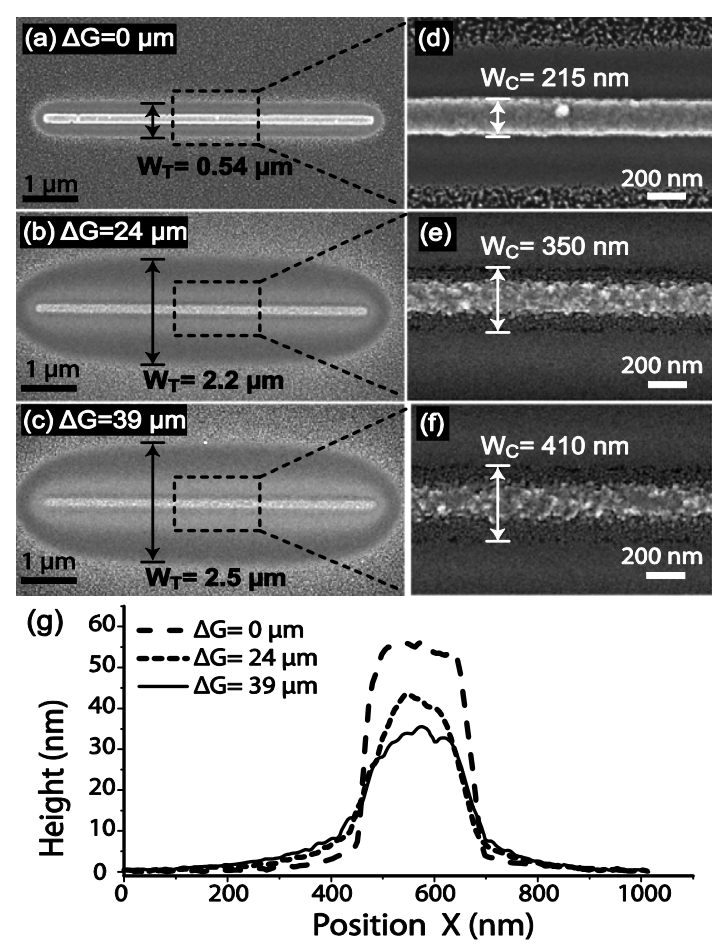

Figure 5. Blurring for different gaps. (a)-(f) SEM images (after contrast etching) of Al structures deposited through $200 \mathrm{~nm}$ wide apertures at different $\Delta G$ values of 0,24 and $39 \mu \mathrm{m}$ using a stair-stencil. As the gap increases, the halo increases ((a)-(c)) and the central structures get more blurred ((d)-(f)). (g) Profiles from TM AFM analysis (before contrast etching). The structures are broader and thinner as the gap increases.

$\alpha=0.0048$ and $\beta=20 \mathrm{~nm}$. The halo-blurring, $B_{\mathrm{H}}$, is plotted versus $\Delta G$ in figure $6(\mathrm{~b}) . B_{\mathrm{H}}$ increases with the gap, and with the aperture width $(A)$ as well. The slope of $B_{\mathrm{H}}$ versus $\Delta G$ is largest for $A=1 \mu \mathrm{m}$ and smallest for $A=100 \mathrm{~nm}$. The slope of $B_{\mathrm{H}}$ versus $\Delta G$ is not constant, it decreases for $\Delta G \gtrsim$ $25 \mu \mathrm{m}$. Images of deposited structures showing the blurring for different aperture sizes are shown in the supporting information in figure SI 3 (available at stacks.iop.org/Nano/20/415303). The thickness of the structures is plotted in figure 6(c). Starting from the nominal value of $60 \mathrm{~nm}$, the thickness decreases for larger gaps and smaller apertures. This behavior has been previously observed for $\mathrm{Au}$ and $\mathrm{Al}$ nanowires deposited through stencils [6].

\subsection{Effect of deposition parameters}

2.4.1. Nominal deposition thickness. Different evaporations changing the nominal deposition thickness of aluminum were performed on a silicon substrate using the slope-stencils described before in section 2.2. The nominal deposition thickness corresponds to the value registered by the measuring crystal quartz of the evaporator. Different nominal thicknesses of 20,60 and $100 \mathrm{~nm}$ of $\mathrm{Al}$ were deposited at $4 \AA \mathrm{s}^{-1}$ $\left(10^{-6}\right.$ mbar, $300 \mathrm{~K}$ and $\left.D=1 \mathrm{~m}\right)$ using the same stencil. In order to avoid clogging problems, we removed the $\mathrm{Al}$ from the stencil after each deposition [20]. The results for a deposition through a $600 \mathrm{~nm}$ wide aperture are shown in 

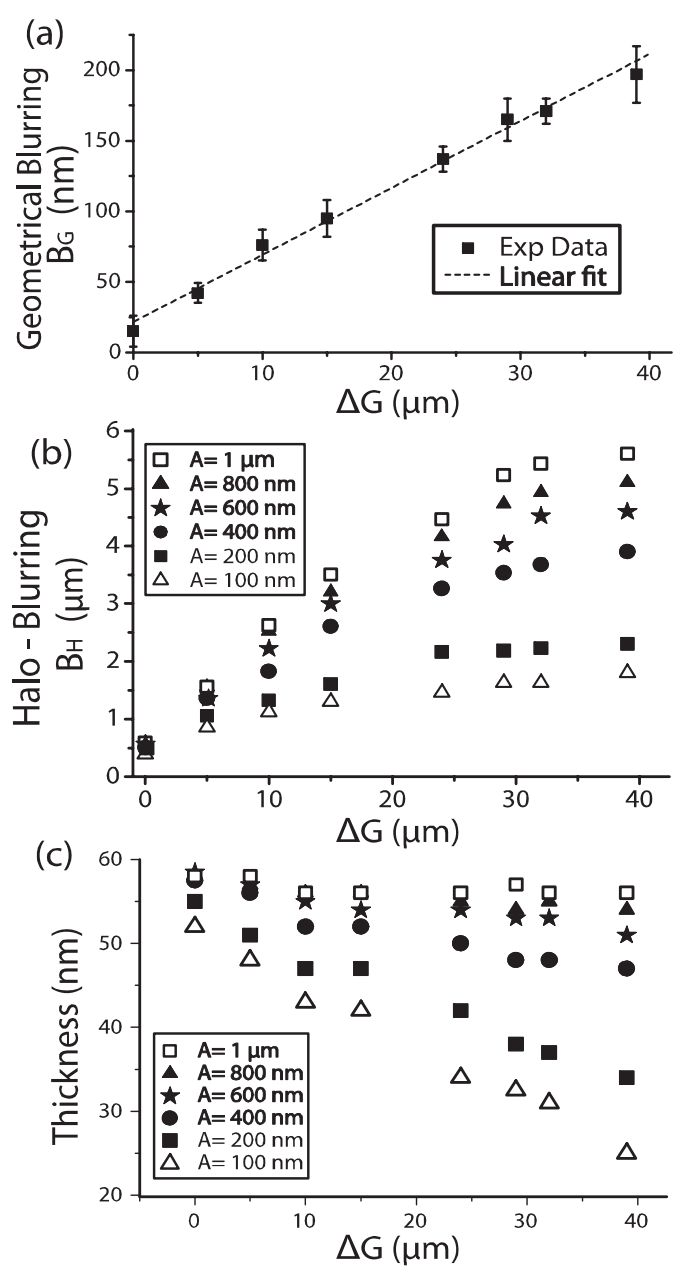

Figure 6. (a) Geometrical blurring, $B_{\mathrm{G}}$, as a function of $\Delta G$. The dotted line corresponds to a linear fit. (b) Halo-blurring, $B_{\mathrm{H}}$, as a function of $\Delta G$ for different aperture widths. $B_{\mathrm{H}}$ increases for larger gaps and wider apertures. (c) Structure thickness as a function of $\Delta G$ for different aperture sizes $A$. The thickness is reduced as the gap increases and the aperture size is reduced. (Data obtained by a deposition of $60 \mathrm{~nm}$ thick aluminum through a stair-stencil.)

figure 7, showing an increase in the halo as the thickness is increased. The left part of the structures had the smallest stencil-substrate gap, increasing gradually towards the right extreme (see figure 4(b)). These depositions also confirm the increase in the halo as the gap increases. It is also important to remark that the blurring for a specific point is due not only to its corresponding gap, but also due to contributions of neighbor sections with different gaps. In figure 7 it is observed that the two extremes of the structures have smaller halos since there is less material coming from neighbor zones compared with sections in the middle of the structure.

2.4.2. Deposition rate. The effect of the deposition rate on the blurring was also analyzed. Two depositions of $45 \mathrm{~nm}$ of $\mathrm{Al}$ were done through the same apertures using a slope-stencil $\left(10^{-6} \mathrm{mbar}, 300 \mathrm{~K}\right.$ and source-substrate distance $\left.D=1 \mathrm{~m}\right)$. One deposition was done at $1 \AA \mathrm{s}^{-1}$ and the second at $4 \AA \mathrm{s}^{-1}$. The results are illustrated in figures $8(a)$ and (b) for structures deposited through a $300 \mathrm{~nm}$ wide aperture, showing a larger blurring at $4 \AA \mathrm{s}^{-1}$ than at $1 \AA \mathrm{s}^{-1}$. As observed, the halo is
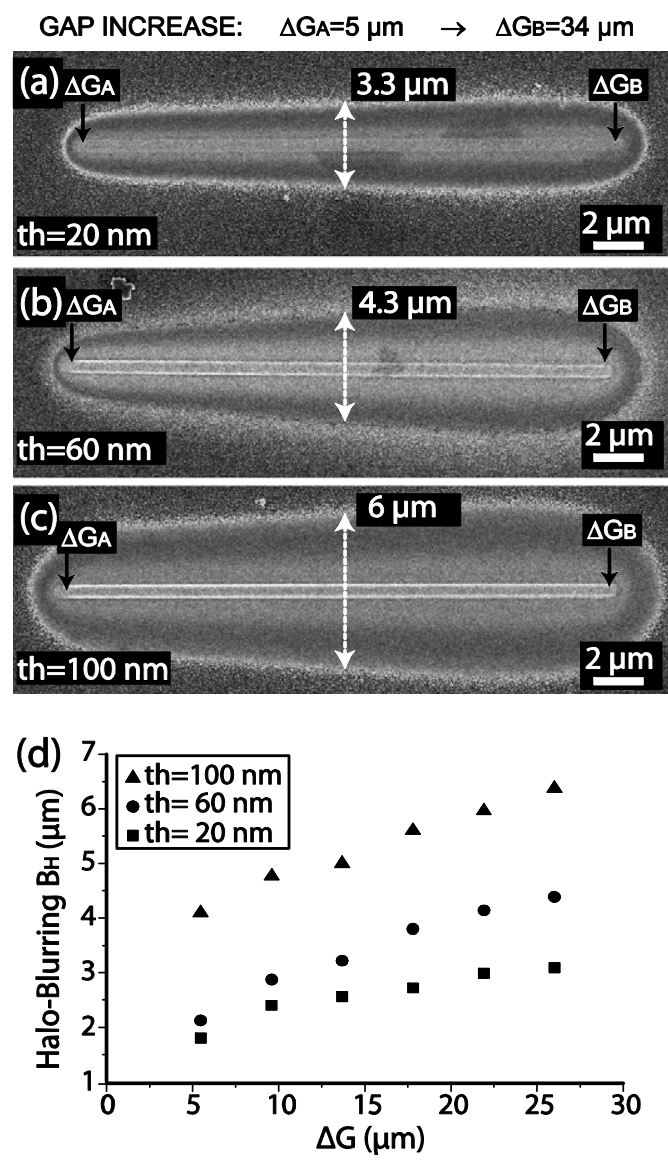

Figure 7. SEM images (after contrast etching) of depositions for different nominal thickness of (a) 20, (b) 60, and (c) $100 \mathrm{~nm}$ of $\mathrm{Al}$ through the same aperture $(A=600 \mathrm{~nm})$ using a slope-stencil. The change from the smallest (left) to the largest (right) gap is $\sim 30 \mu \mathrm{m}$ (see figure 4(b)). For larger nominal thicknesses the halo becomes larger. As the gap increases, the size of the halo increases as well. (d) Plot of $B_{\mathrm{H}}$ versus $\Delta G$ for the structures shown in (a)-(c).

reduced and the definition of the central structure is improved for the slower deposition rate. This is confirmed by the TM AFM profile of the structures (before contrast etching) shown in figure 8(c). The structure deposited at $4 \AA \mathrm{s}^{-1}$ has a broader and thinner profile compared to the one at $1 \AA \mathrm{s}^{-1}$. In order to confirm this behavior, the same experiment was performed using a different evaporator with a source-substrate distance $D=50 \mathrm{~cm}$. Three depositions of $45 \mathrm{~nm}$ of $\mathrm{Al}$ were done at 2, 10 and $40 \AA \mathrm{s}^{-1}$. The results showed the same trend obtained before. The deposition done at $2 \AA \mathrm{s}^{-1}$ had the least blurring and the one at $40 \AA^{-1}$ the largest. The structures obtained from these three depositions are shown in the supporting information in figure SI 4 (available at stacks.iop.org/Nano/20/415303). Figure 8(d) shows the results for the halo-blurring as a function of the gap for the different deposition rates in the two different evaporators with sourcesubstrate distances and aperture widths of $\left(D_{1}=1 \mathrm{~m}, A_{1}=\right.$ $300 \mathrm{~nm})$ and $\left(D_{2}=50 \mathrm{~cm}, A_{2}=650 \mathrm{~nm}\right)$. These results show that the halo-blurring increases for faster deposition rates.

2.4.3. Substrate temperature. Finally, we also studied the effect of the substrate temperature on the blurring. Two 

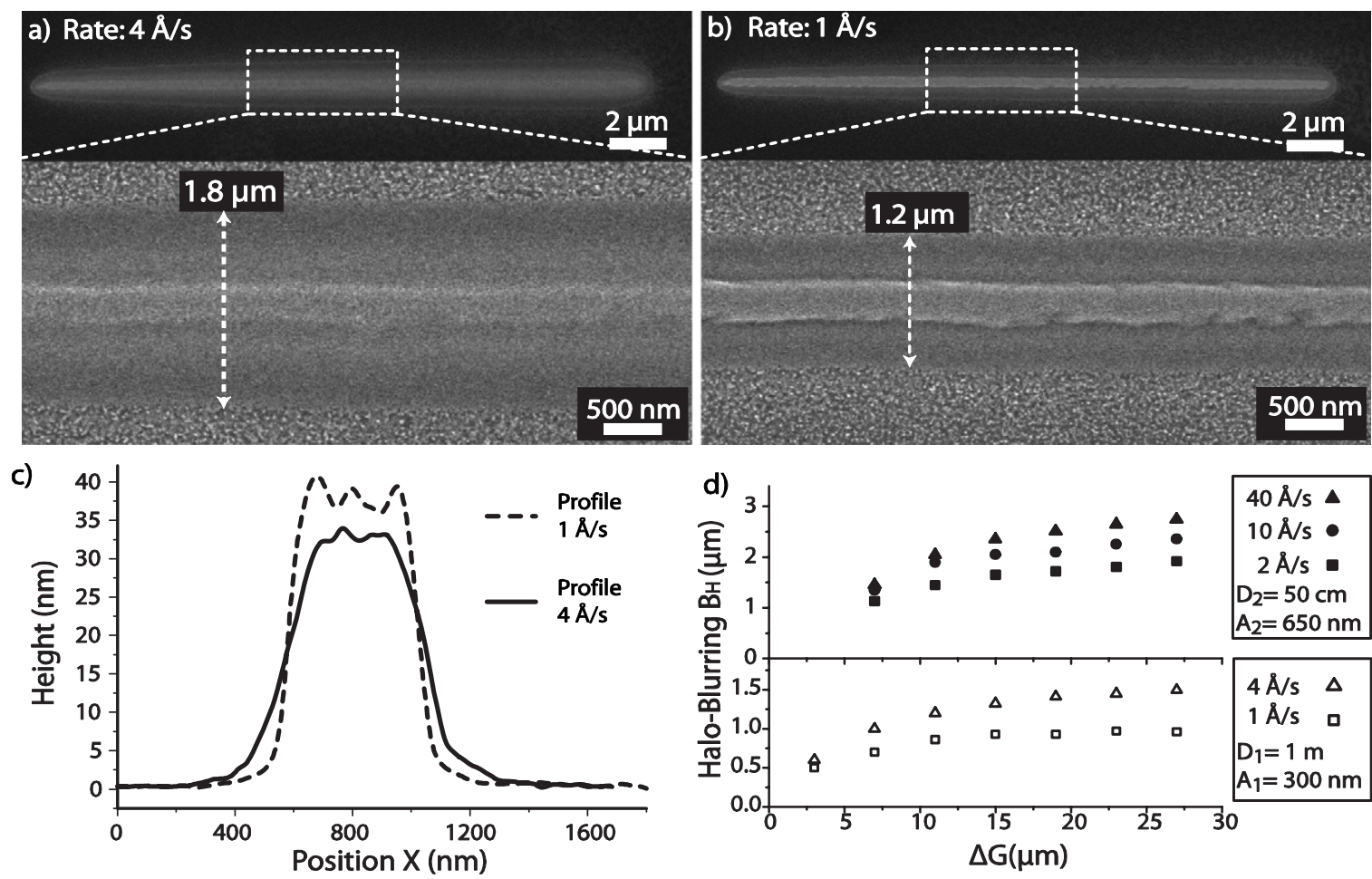

Figure 8. Blurring for different deposition rates. SEM images of Al structures deposited at (a) $4 \AA \mathrm{s}^{-1}$ and (b) $1 \AA \mathrm{s}^{-1}$ through the same aperture with a slope-stencil. The gap variation is $\sim 30 \mu \mathrm{m}$ from the left to the right extremes of the structures. The nominal deposition thickness is $45 \mathrm{~nm}$ and the aperture width is $300 \mathrm{~nm}$. For the slower deposition rate of $1 \AA \mathrm{s}^{-1}$, the size of the halo decreases and the central structure is better defined. (c) TM AFM profiles (before contrast etching) showing a broader and thinner profile for the structure deposited at $4 \AA \mathrm{s} \mathrm{s}^{-1}$. (d) $B_{\mathrm{H}}$ as a function of $\Delta G$ for different deposition rates using two different evaporators.

evaporations of $40 \mathrm{~nm}$ thick $\mathrm{Al}$ were performed at substrate temperatures of $-100^{\circ} \mathrm{C}(173 \mathrm{~K})$ and $115^{\circ} \mathrm{C}(388 \mathrm{~K})$. In this case, the evaporations were done by thermal PVD, with a source-substrate distance $D=20 \mathrm{~cm}$, a deposition rate of $\sim 5 \AA \mathrm{s}^{-1}$ and using stencils with conventional flat membranes. Figure 9 illustrates the results for structures deposited through $500 \mathrm{~nm}$ wide apertures after the contrast etching. Regarding the halo, there is no significant difference between the two depositions at different temperatures. However, an important difference is observed concerning the central structure. Even though they have a similar size, the central structure in the deposition at lower temperature is better defined, showing a clearer edge. The grain size of the central structure is also larger for the higher temperature deposition.

\subsection{Blurring for different metals}

Even though the previous analyses were done only for $\mathrm{Al}$ depositions, a similar behavior for the blurring can be observed for other metals. Figure 10 shows structures of different metals, Al, Au, Pt, Ti and $\mathrm{Cr}$, deposited at $4 \AA \mathrm{s}^{-1}$ through apertures in slope-stencils on a silicon substrate after a contrast etching, except for $\mathrm{Au}$, due to an incompatibility with the dry etching process equipment. For each structure the gap increases gradually from the left to right extremes. In a similar way to $\mathrm{Al}$, for $\mathrm{Pt}$, $\mathrm{Ti}$ and $\mathrm{Cr}$, the deposited structure also consists of a central structure surrounded by a halo that increases with the gap. In the case of $\mathrm{Au}$, the deposited structure gets broader as the gap increases, but it is not possible to observe the halo or determine its behavior. These results suggest that the halo behavior is common for metallic structures deposited through stencils. However, a comparison of the blurring between the materials presented requires further analysis because the depositions were not all done with the same stencils, the deposition thickness and source size are not the same, and the selectivity to the contrast etching can also be different.

\section{Discussion}

\subsection{Effect of geometry on blurring}

In PVD by evaporation, the flow of evaporated material has a characteristic cosine distribution following a line of sight trajectory from the source to the substrate [21]. This behavior is due to the much larger mean free path $\left(l_{o}\right)$ of the vaporized atoms compared with the source-substrate distance $\left(l_{o}>\right.$ $D$ ) [21]. In our conditions, at $P=10^{-6}$ mbar and $T=300 \mathrm{~K}$, the mean free path for atomic particles (diameter $\sim 2 \AA$ ) is $l_{o} \sim 100 \mathrm{~m}$, satisfying $l_{o} \gg D$. This condition is the basis for the geometrical blurring model (figure 1 and equations (2) and (3)). Comparing the results of the linear fit for the measured geometrical gap (figure 6(a)), with the model for $B_{\mathrm{G}}$ of equation (3),

$$
B_{\mathrm{G}}=\frac{\left(G_{o}+\Delta G\right) S}{D}=20 \mathrm{~nm}+0.00485 \Delta G
$$



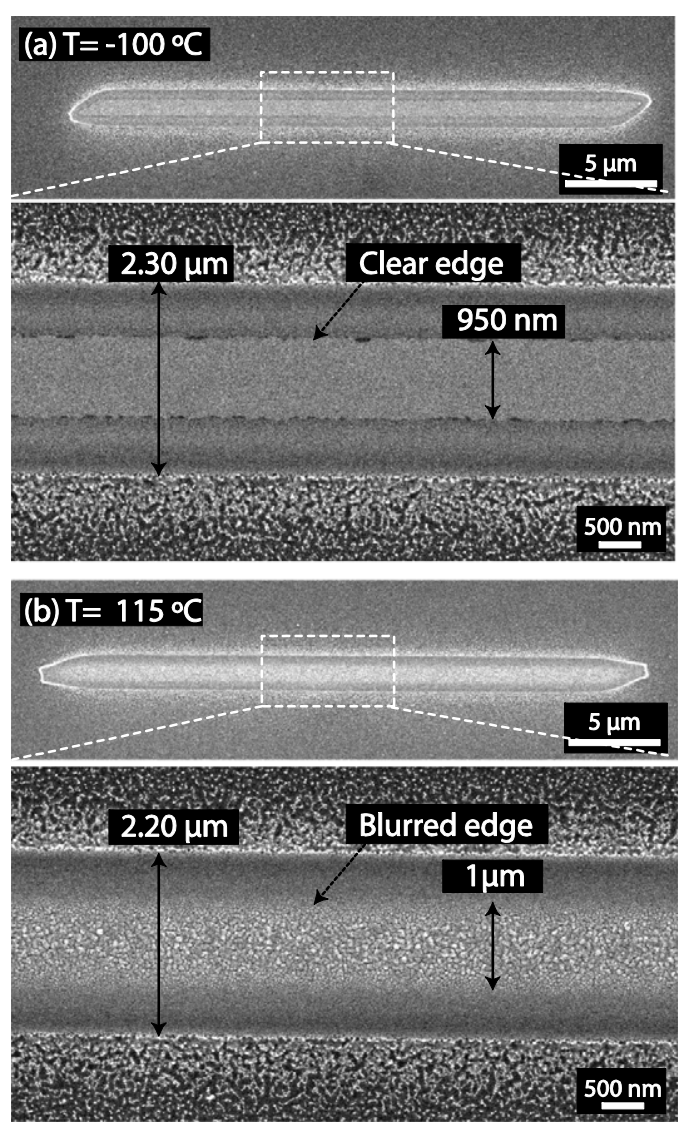

Figure 9. Blurring for different substrate temperatures. SEM images of depositions at (a) $-100^{\circ} \mathrm{C}$ and (b) $115^{\circ} \mathrm{C}$ after a contrast etching. There is no significant change in the size of the halo and the central structure. However, the structure deposited at higher temperature has a more blurred edge in the central structure and a larger grain size.

we can extract $S=0.0048 \mathrm{~m}$ and $G_{o}=4.1 \mu \mathrm{m}(D=1 \mathrm{~m}$ is known). The value for $S$ is reasonable, since the spot size of the electron beam heating the source is $\sim 5 \mathrm{~mm}$ without any e-beam sweeping. The real size of the source is difficult to estimate, since the actual area that evaporates depends on the power, size and sweep geometry of the e-beam and on the thermal properties of the material to evaporate. The value extracted for $G_{o}$ is also close to the measured value by optical microscopy of $\sim 3 \mu \mathrm{m}$, and in the range of the typical values $\left(G_{o}<\sim 5 \mu \mathrm{m}\right)$ observed in this configuration. These results support the validity of equation (3) to describe the geometrical blurring and to estimate the size of the central structures for depositions through stencils by evaporation PVD.

Rather than a profile of the deposited structure, the geometrical blurring model gives a profile of the flux of material impinging on the substrate. From geometrical considerations, it is possible to distinguish between two different zones on the substrate. As illustrated in figure 11(a), there is a central zone $Y$ under the aperture that receives material from the entire source with a maximal deposition rate, and two side zones $X$ that receive material only from a fraction of the source, having a reduced deposition rate. The deposition rate decreases starting from the edge of the $Y$ zone and becomes zero at the outer edges of the $X$ zones. However,
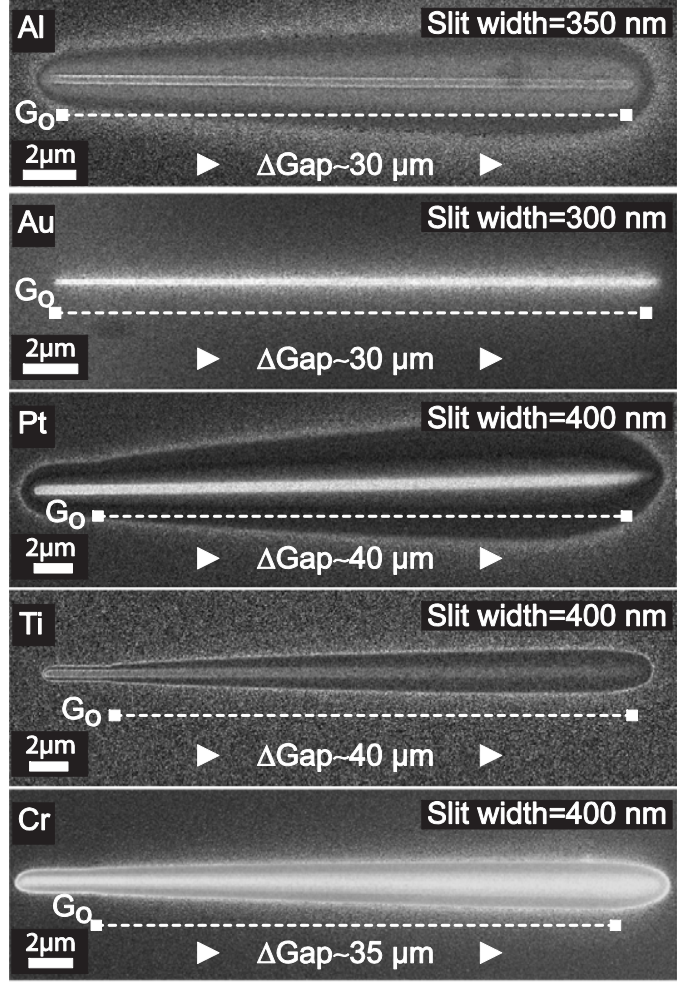

Figure 10. Blurring for different metals. SEM images of different metals deposited through slope-stencils. The metals deposited and their thickness are: aluminum $(60 \mathrm{~nm})$, gold $(60 \mathrm{~nm})$, platinum $(40 \mathrm{~nm})$, titanium $(40 \mathrm{~nm})$ and chrome $(25 \mathrm{~nm})$. Except for gold, a contrast etching was done. The gap increases from left to right and the change in gap ( $\Delta$ Gap) is indicated in each case. The increase in halo with the gap is clear for the different metals, except for Au, for which the halo is not clearly observed.

if the gap increases or the aperture size decreases such that $G / A>D / S$, then even the central zone $Z$ under the aperture can 'see' only a fraction of the source (figure 11(b)). This reduction in the effective size of the source reduces the local deposition rate and hence the thickness of the entire structure. This probably contributes to the reduction of the thickness of the deposited structures for large gaps and small apertures observed in the results of figure 6(c).

\subsection{Spreading of material after adsorption}

The geometrical blurring cannot be an exact model for the blurring because it does not consider the dynamics of the adsorbed molecules on the substrate. As described before, the structures deposited by SL have a central structure surrounded by a thin halo. The halo and the material spreading have been previously observed for shadow mask depositions by PVD [22, 18, 16, 23, 24]. Rácz et al observed a halo between 0.1 and $2 \mu \mathrm{m}$ for different metals deposited through apertures of 3-7 $\mu \mathrm{m}$ in size by e-beam evaporation on substrates at $45^{\circ} \mathrm{C}$ [18]. They also reported that the smallest halo-blurring was observed for $\mathrm{Ge}$ and $\mathrm{Cr}$ depositions and the largest for $\mathrm{Al}$, Ti and Pt. Levenson et al also observed a spreading of material varying between 8 and $25 \mu \mathrm{m}$ using a $50 \mu \mathrm{m}$ diameter wire as 


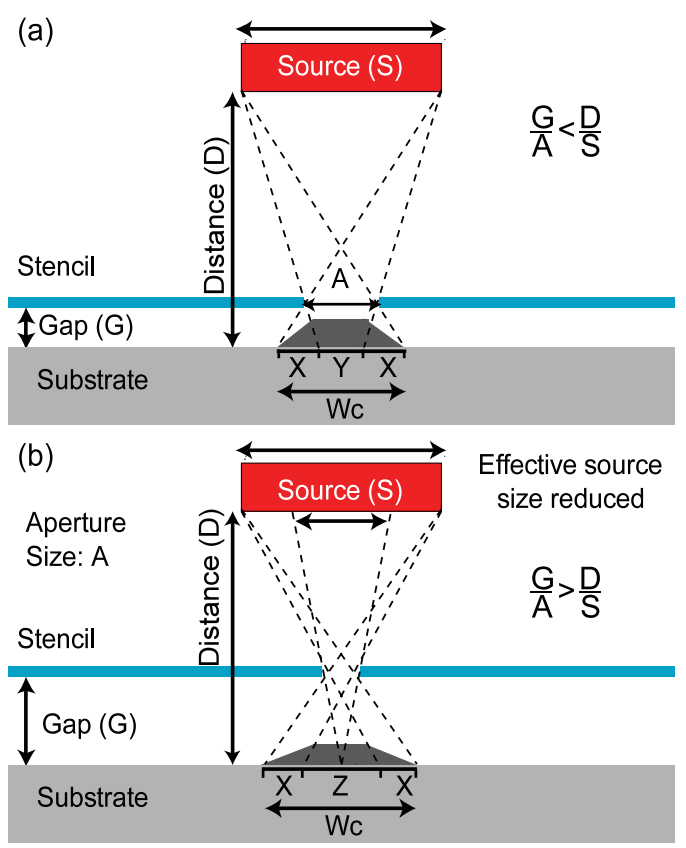

Figure 11. Material flux profile. (a) Scheme illustrating the central $Y$ zone receiving material from the entire source and the side $X$ zones receiving material from a fraction of the source when $G / A<D / S$. (b) For larger gaps or smaller apertures such that $G / A>D / S$, the zone $Z$ under the aperture 'sees' only a fraction of the source, reducing the final thickness. (Halo not illustrated.)

a shadow mask for $\mathrm{Al}$ depositions on oxides and silicon nitride substrates at $100{ }^{\circ} \mathrm{C}[22]$.

The formation of the halo is probably related to the atomistic processes involved in film growth. The deposition of a structure by evaporation PVD implies the condensation of the atoms on the substrate, heating the substrate a few degrees $\left(1-10^{\circ} \mathrm{C}\right)$ for a conventional silicon wafer $[25,26,21]$. The growth of the structures is driven by both thermodynamics and kinetics. When the atoms arrive to the surface, they remain mobile due to surface diffusion and can join an existing island, form a new nucleation center or eventually be desorbed [27, 28]. The diffusion of atoms during film growth is driven by a gradient in the chemical potential and is a thermally activated process. The surface diffusion activation energy $E_{\mathrm{d}}$ and the diffusion coefficient $D=$ $D_{o} \exp \left(-E_{\mathrm{d}} / k_{\mathrm{B}} T\right)$ are not fixed constants and may depend on the adatom coverage and the adatom-adatom and adatomsubstrate interactions [29, 30]. The activation energy for diffusion of metallic atoms on metals is $E_{\mathrm{d}} \sim 0.5-1 \mathrm{eV}$, which is normally a fraction between $1 / 2$ and $1 / 4$ of the adsorption energy $E_{\mathrm{a}}$ (typically $E_{\mathrm{a}}>1 \mathrm{eV}$ ). The parameter $D_{o}$ for metals is typically in the order of $\sim 10^{-7} \mathrm{~m}^{2} \mathrm{~s}^{-1}$ [31]. (For a more detailed expression of the diffusion coefficient see the supporting information in section 4.) These different phenomena and factors, like nucleation, diffusion, substrate properties and deposition conditions, affect the dynamics of the adsorbed atoms and probably determine the formation of the halo.

In the early stages of condensation and growth, the adsorbed atoms diffuse and bind to other atoms, forming nucleation centers and stable islands. As the islands grow in size, most of the incoming atoms join existing islands, generating the steady growth of a condensed structure [28]. This is probably the case for the areas on the substrates on zones $Y$ and $Z$, and parts of zones $X$ (see figure 11), generating the well defined central structure. However, in the outer edges of zones $X$, the landing atoms can diffuse out of the deposition zone, where the probabilities to find a stable grain or nucleate with another atom are much lower, increasing the diffusion time and length. This could produce a thin layer of spread material, e.g. the halo, surrounding the well defined central structure. Another difference between the atoms forming the halo with those forming the central structure is the interaction with the substrate. After a few seconds (1$2 \mathrm{~nm}$ thick deposition), a complete metallic layer is formed under the stencil aperture (mainly zones $Y$ and $Z$ ), meaning that the after-coming atoms grow on a metallic substrate of the same composition as the condensing material (metallic atoms on metal). On the other hand, the atoms diffusing out of the deposition zone forming the halo, diffuse mainly on the original substrate (metallic atoms on silicon oxide), since in this zone it takes more time to form a complete metallic layer. This difference in substrate interaction can also generate different diffusion and adsorption behaviors between the halo zone and the zones under the apertures. From the size of the halo, we can make a rough estimation of a diffusion length of $\sim 1 \mu \mathrm{m}$, and taking as time reference the deposition time around $\sim 100 \mathrm{~s}$, we estimate a diffusion constant $D=$ $\left(1 \mu \mathrm{m}^{2} / 100 \mathrm{~s}\right)=10^{-14} \mathrm{~m}^{2} \mathrm{~s}^{-1}$ and $E_{\mathrm{d}}=0.4 \mathrm{eV}$ assuming $T \sim 300 \mathrm{~K}$. This is comparable to the reported values of 0.4 and $0.9 \mathrm{eV}$ for the diffusion activation energy of $\mathrm{Al}[32,33]$. More detailed analysis and experiments of the halo dependence on temperature and materials would provide more information to determine the role of diffusion in the formation of the halo.

\subsection{Halo dependence on gap and deposition parameters}

As discussed in section 3.1, the geometry of the sourcestencil-substrate configuration affects the profile of material flux impinging the substrate and thus the local deposition rate. The deposition rate is an important parameter affecting the nucleation rate, the growth of the structure and heating phenomena. Since all these elements affect the halo formation, it is expected that the stencil-substrate gap has an effect on the halo. As the gap increases, there are larger zones with a reduced deposition rate (outer edges of zones $X$ ), where the formation of islands is reduced. This increases the amount of atoms that can diffuse and spread out of the deposition zone, forming a larger halo. It is important to note that even for very small gaps, the material can always diffuse laterally since there are no barriers limiting its movement. From the value of $G_{o}$ extracted in section 3.1 for the data in figure 6(a), it is possible to estimate the values of $B_{\mathrm{H}}$ for $G \rightarrow 0$ assuming $\Delta G=-4.1 \mu \mathrm{m}$ in the plot in figure 6(b). This gives values for $B_{\mathrm{H}}$ below $200 \mathrm{~nm}$ for the different aperture sizes. These values could be further reduced optimizing the deposition parameters. However, further analysis and experimental data is required to establish an analytic and reliable relation between the halo and the gap. 
The increase of the halo with the nominal deposition thickness could be due to more material arriving on the substrate spreading and diffusing out to form the halo. This behavior was also observed by Racz et al for different metals [18]. Concerning the effect of the substrate temperature, it is worth to point out the results from Levenson et al and Doust et al [22, 24]. In the work from Levenson, they performed $375 \mathrm{~nm}$ thick $\mathrm{Al}$ depositions and found a maximum size for the halo at $\sim 200^{\circ} \mathrm{C}$ and a reduced halo at 100 and $30{ }^{\circ} \mathrm{C}$ [22]. Similarly, Doust et al performed evaporations of $\mathrm{Ag}$ on $\mathrm{Si}(111)$ substrates in ultrahigh vacuum and observed the maximum spreading at $470{ }^{\circ} \mathrm{C}$ and a reduced spreading for lower or higher temperatures [24]. Raynard et al proposed a model for this behavior based on diffusion and rate equations of thermally activated processes [34]. In their model, the spreading of material decreases for lower temperatures due to a lower diffusion coefficient, whereas for higher temperatures the reduction in the residence time (faster desorption) of the adatoms limits the spreading of the material. This behavior can also be present in our experiments, and the maximum halo size could be found effectively in the range between -100 and $115^{\circ} \mathrm{C}$. The effect of the deposition rate on the halo has also been studied by Racz et al, however, they observed an opposite trend to ours [18]. In their work, they have deposited 5, 10 and $20 \mathrm{~nm}$ of $\mathrm{Al}$ at 0.8 and $2.3 \mathrm{~nm} \mathrm{~s}^{-1}$, through $3-7 \mu \mathrm{m}$ wide square apertures. They have observed a smaller halo for the deposition at $2.3 \mathrm{~nm} \mathrm{~s}^{-1}$ than at $0.8 \mathrm{~nm} \mathrm{~s}^{-1}$. Unfortunately, they do not report the gap values, which also affect the size of the halo. From the surface diffusion point of view, at higher deposition rates the formation of islands is faster, leading to smaller diffusion lengths. However, the higher deposition rate also leads to higher heating of the substrate and hence to a higher diffusion coefficient. In the halo zone, where the nucleation is reduced, the increase in the surface diffusion coefficient can become a dominant factor and lead to a larger halo. More experiments varying the temperature and deposition rate would be important to understand this behavior and their effect on atomic dynamics and surface diffusion in SL.

\subsection{Solutions for halo-blurring}

As a remedy for the halo, Arcamone et al developed a corrective etching that exploits the reduced thickness of the halo [23]. It consists of an anisotropic dry etching of the deposited material of a few nanometers, 1-3 nm, enough to remove the halo while leaving the central structure clearly defined. However, its application is limited to the compatibility with the other materials and fabrication processes involved.

The blurring can also be reduced by adjusting the deposition parameters and/or by reducing the gap between the stencil and the substrate. The gap depends strongly on the wafer curvature and on the clamping and fixation of the stencil to the substrate. Normally smaller stencils $(<1 \mathrm{~cm})$ reduce the effect of the curvature and produce gaps $<5 \mu \mathrm{m}$. Similarly, different clamping systems result in different gaps. Clamping systems that provide a uniform force over the entire stencil and substrate normally result in smaller and more uniform gaps.
A reduction in the gap can also be achieved using compliant membranes that would follow the topography of the substrate, reducing the gap and improving the definition of the deposited structures [35].

\section{Conclusions}

We have shown that the blurring of structures deposited by stencil lithography depends on the source-stencil-substrate geometrical configuration and on the deposition parameters. The blurring is manifested as an enlargement of the structures and as a halo, both increasing when the stencil-substrate gap increases. The size of the central structure shows a linear behavior with respect to the gap, which proves that the geometrical model for the blurring gives a good approximation for the size of structures deposited through stencils. The halo surrounding the structure increases not only with the gap, but also with the stencil aperture size. More experiments and analysis are required to establish an analytical relation between these factors. We have also demonstrated that other deposition parameters like the thickness, deposition rate and substrate temperature affect the blurring, highlighting the role of atomic dynamics and surface diffusion in the blurring in stencil lithography. These results show that by reducing the gap and controlling the deposition parameters, it is possible to reduce the blurring and increase the resolution of stencil lithography. However, the understanding of the blurring, the halo and the role of surface diffusion still requires further analysis that would provide clearer strategies to reduce the blurring.

\section{Experimental details}

Stencil fabrication. The details of the fabrication process are given in the supporting information (available at stacks.iop.org/Nano/20/415303). The fabrication is based on silicon-based micromachining. The profiles of the stencils are defined by deep reaction ion etching for the stair-stencils and by anisotropic $\mathrm{KOH}$ etching for the slope-stencils. The nanoapertures are defined with a focused ion beam.

Contrast etching. The contrast etching is based on a silicon dry anisotropic etching using $\mathrm{SF}_{6}$ and $\mathrm{CF}_{4}$ with an Alcatel 601 equipment. The Si etching rate is $400 \mathrm{~nm} \mathrm{~min}^{-1}$, with a selectivity to aluminum of $\sim 100$, and performed during $10 \mathrm{~s}$ (Si etch depth of $\sim 60 \mathrm{~nm}$ ).

Evaporators. Most of the experiments were carried out in an electron beam evaporator Leybold LAB600 $(D=1 \mathrm{~m})$. The machine is operated in automatic mode, with a feedback loop to control the deposition rate. For the experiments with different deposition rates, we also used an electron beam evaporator Alcatel EVA600 $(D=40 \mathrm{~cm})$ with a manual deposition rate control. The experiments with substrates at different temperatures were done on an Edwards Auto 306 thermal evaporator with a source-substrate distance of $20 \mathrm{~cm}$ and home-made substrate temperature control. 


\section{Acknowledgments}

This effort was sponsored by the Swiss Federal Office for Science and Education (OFES) in the EC-funded FP6 project NaPa (NMP4-CT-2003-500120), the Swiss National Science Foundation (IC-Nano-200021-112291/1) and the EPFL-STI Seed Funding. The authors are very grateful to the EPFLCMI and its staff, especially to Dr Philippe Fluckiger and Guy Clerc for their effort implementing stencil lithography. We are also grateful to Dr. Cristina Martin for her help with AFM analysis and to Michel Schär and Professor L Zuppiroli for the depositions at different substrate temperatures.

\section{References}

[1] Kohler J, Albrecht M, Musil C R and Bucher E 1999 Physica E 4196

[2] Deshmukh M M, Ralph D C, Thomas M and Silcox J 1999 Appl. Phys. Lett. 751631

[3] Brugger J, Berenschot J W, Kuiper S, Nijdam W, Otter B and Elwenspoek M 2000 Microelectron. Eng. 53403

[4] Yan X M, Contreras A M, Koebel M M, Liddle J A and Somorjai G A 2005 Nano Lett. 51129

[5] Tun T N, Lwin M, Kim A H H, Chandrasekhar A N and Joachim C 2007 Nanotechnology 18335301

[6] Vazquez-Mena O, Villanueva G, Savu V, Sidler K, van den Boogaart M A F and Brugger J 2008 Nano Lett. 83675

[7] Savu V, Neuser S and Brugger J 2008 MNE'08 (Athens)

[8] Arcamone J, Boogaart M A F v d, Serra-Graells F, Fraxedas J, Brugger J and Perez-Murano F 2008 Nanotechnology 19305302

[9] Speets E A, Riele P T, van den Boogaart M A F, Doeswijk L M, Ravoo B J, Rijnders G, Brugger J, Reinhoudt D N and Blank D H A 2006 Adv. Funct. Mater. 161337

[10] Zhou Y X, Johnson A T, Hone J and Smith W F 2003 Nano Lett. 31371

[11] Sidler K, Vazquez-Mena O, Savu V, Villanueva G, van den Boogaart M A F and Brugger J 2008 Microelectron. Eng. 851108

[12] Villanueva G, Vazquez-Mena O, van den Boogaart M A F, Sidler K, Pataky K, Savu V and Brugger J 2008 Microelectron. Eng. 851010
[13] Pang S W, Geis M W, Goodhue W D, Efremow N N, Ehrlich D J, Goodman R B and Randall J N 1988 J. Vac. Sci. Technol. B 6249

[14] Villanueva G, Martin C, Vazquez-Mena O, Montserrat J, Langlet P, Bausells J and Brugger J 2008 MNE'08 (Athens)

[15] Lishchynska M, Bourenkov V, van den Boogaart M A F, Doeswijk L, Brugger J and Greer J C 2007 Microelectron. Eng. 8442

[16] Tiggelaar R M, Berenschot J W, Elwenspoek M C, Gardeniers J G E, Dorsman R and Kleijn C R 2007 J. Vac. Sci. Technol. B 251207

[17] Arcamone J, Sansa M, Verd J, Uranga A, Abadal G, Barniol N, van den Boogaart M, Brugger J and Pérez-Murano F 2009 Small 5176

[18] Racz Z and Seabaugh A 2007 J. Vac. Sci. Technol. B 25857

[19] Horcas I, Fernandez R, Gomez-Rodriguez J M, Colchero J, Gomez-Herrero J and Baro A M 2007 Rev. Sci. Instrum. 78013705

[20] Vázquez-Mena O, Villanueva G, van den Boogaart M A F, Savu V and Brugger J 2008 Microelectron. Eng. 851237

[21] Mahan J E 2000 Physical Vapor Deposition of Thin Films (New York: Wiley-Interscience)

[22] Levenson L L, Swartzlander A B, Yahashi A, Usui H and Yamada I 1990 J. Vac. Sci. Technol. A 81447

[23] Arcamone J, Sanchez-Amores A, Montserrat J, van den Boogaart M A F, Brugger J and Perez-Murano F 2007 J. Micro-Nanolithogr. MEMS MOEMS 6013005

[24] Doust T, Metcalfe F L and Venables J A 1989 Ultramicroscopy 31116

[25] Breitweiser G, Varadarajan B N and Wafer J 1970 J. Vac. Sci. Technol. 7274

[26] Krasovskii A M, Palii O I and Rogachev A V 1977 Mech. Compos. Mater. 1347

[27] Zinsmeister G 1966 Vacuum 16529

[28] Zhang Z and Lagally M G 1997 Science 276377

[29] Ehrlich G and Stolt K 1980 Annu. Rev. Phys. Chem. 31603

[30] Gomer R 1990 Rep. Prog. Phys. 53917

[31] Bonzel H P 2006 Surface diffusion on metals Diffusion in Solid Metals and Alloys vol 26, ed H Mehrer (Berlin: Springer)

[32] Liu C L, Cohen J M, Adams J B and Voter A F 1991 Surf. Sci. 253334

[33] Grabowski S, Kadau K and Entel P 2002 Phase Transit. 75265

[34] Raynerd G, Doust T N and Venables J A 1992 Surf. Sci. 261251

[35] Sidler K, Villanueva G, Vazquez-Mena O and Brugger J 2009 Digest of Technical Papers-Transducers'09 p 1612 